\title{
Interplay Between the Active Phase and Support: Preparation, Characterization and Catalytic Performance
}

\author{
Dmitry Yu. Murzin
}

Published online: 13 January 2009

(C) Springer Science+Business Media, LLC 2009

Metal nanoparticles formed in various media and stabilized by different mechanism are the objects of intense study in contemporary catalytic research due to their unique properties, which are significantly influenced not only by the size of nanoparticles and their surfaces, but by the chemical nature of the environment surrounding such nanoparticles. While the main attention is focused on the properties of metal nanoparticles per se, the effect of the matrix on the metal properties is not very well understood, especially when comparing inorganic and organic matrices having the same metal nanoparticle sizes. At the same time the local metal environment, which can be influenced during catalyst preparation for example by catalyst pretreatment or modification of support acidity, etc., may affect both the metal particle size and shape/morphology of the catalysts.
This issue contains a collection of papers describing synthesis of nanosized $\mathrm{Ru}, \mathrm{Pt}, \mathrm{Au}$ and $\mathrm{Pd}$ metal catalysts supported on inorganic and organic matrices (inorganic micro-mesoporous matrices, carbon nanofibers or polymer based materials) as well as their characterization and testing in industrially important reactions for producing mainly fine and specialty chemicals.

We consider this collection of articles, which are written by participants in an EU funded research project NANOCAT (No. 506621), to be a valuable addition to the literature on interactions between nanosized metal and supports of varying chemical nature.

D. Yu. Murzin $(\bowtie)$

Åbo Akademi University, Biskopsgatan 8, Åbo/Turku 20500,

Finland

e-mail:dmurzin@abo.fi 